\title{
Editorial Errata
}

Published online: 18 May 2012

(C) Springer Science+Business Media, LLC. 2012

The PIL special issue on Malingering [part II, published in June 2011, vol. 4(2)], edited by David T. R. Berry and Nathaniel W. Nelson, included an article by Mark D. Allen, Trevor ChuangKuo Wu, and Erin D. Bigler, entitled, Traumatic Brain Injury alters Word Memory Performance by slowing response time and increasing cortical activation: An fMRI study of a Symptom Validity Test (pp. 140-146). The Table of Contents for the issue should have listed this article as a separate one because the Traumatic Brain Injury section of the journal had accepted it for publication. The Editor in Chief (EIC) had included it in the issue Table of Contents as part of the special issue.

The special issue included in particular a series of articles submitted to the Malingering section of the journal. The Editor in Chief had taken the decision to publish the first article as part of the exchange under the journal policy of publishing controversial articles in a response-rebuttal format and letting the reader decide on the merits of the exchange (see R. Rogers, S. C. Bender, \& S. F. Johnson, A critical analysis of the MND criteria for feigned cognitive impairment: Implications for forensic practice and research (pp. 147-156); K. B. Boone, Clarification or confusion? A review of Rogers, Bender, and Johnson's A critical analysis of the
MND criteria for feigned cognitive impairment: Implications for forensic practice and research (pp. 157-162) and R. Rogers, S. C. Bender, \& S. F. Johnson, A commentary on the MND model and the Boone critique: "Saying it doesn't make it so" (pp. 163-167). It should be noted that in all such article response-rebuttal formats in the journal, given the concept that the authors should make their best case for the material published, the authors are responsible for the final content. Once more, the EIC takes full responsibility for publishing this exchange, which he considers timely and valuable.

It should be noted that all authors are requested to declare any conflicts of interest. In the case of the series of articles in the exchange, it has been pointed out that the senior authors involved in the exchange have published forensic tests, which for some might constitute a conflict of interest (COI), although both have tests in their name. Nevertheless, we remind that all publications in the journal should include clear conflict of interest statements by authors. For those who had queried the missing information, once more the EIC takes full responsibility, and in consequence has taken steps to assure that the difficulty is not repeated, such as having section heads to remind contributors of the need for COI consideration. 